\title{
Activation of NR1/NR2B NMDA receptors
}

\author{
Tue G. Banke and Stephen F. Traynelis
}

Emory University School of Medicine, Department of Pharmacology, 1510 Clifton Road, Atlanta, Georgia 30322, USA

Correspondence should be addressed to T.G.B. (tbanke@pharm.emory.edu)

Published online 13 January 2003; doi:10.1038/nn1000

$\mathrm{N}$-methyl-D-aspartate (NMDA) receptors are highly expressed in the central nervous system and are involved in excitatory synaptic transmission as well as synaptic plasticity. Despite considerable structural and biophysical research, the mechanism behind activation of the NMDA receptor is still poorly understood. By analyzing patch clamp recordings of one channel activated by glutamate, we determined the burst structure and open probability for recombinant rat NR1/NR2B receptors. We used partial agonists at the glutamate and glycine binding sites to show that at least two kinetically distinct subunit-associated conformational changes link co-agonist binding to the opening of the NMDA receptor pore. These data suggest that NR1 and NR2B subunits, respectively, undergo a fast and slow agonist-dependent conformational change that precedes opening of the pore. We propose a new working model of receptor activation that can account for macroscopic as well as microscopic NMDA receptor properties.

The NMDA receptor, a member of the family of ionotropic glutamate receptors, is highly expressed in the CNS. NMDA receptors mediate a slow component of synaptic transmission and are involved in synaptic plasticity. Excessive activation of NMDA receptors can contribute to the initiation and propagation of seizures and to the neurodegeneration that accompanies ischemia and stroke ${ }^{1}$. NMDA receptor complexes are comprised of at least four subunits surrounding a central, water-filled pore. Functional receptors contain both NR1 and NR2 subunits, which bind glycine and glutamate, respectively. Co-agonist binding leads to a conformational change in the protein that converts the ion channel to the open or conducting state, and in some instances, to the desensitized state. The time course of the slow NMDA receptor-mediated component of synaptic currents is determined by the single-channel kinetics ${ }^{2}$. Despite intensive study of the macroscopic and microscopic properties of NMDA receptors ${ }^{2-11}$, the mechanism underlying agonistinduced channel opening remains elusive.

To understand the gating of NMDA receptors, we studied single-channel as well as macroscopic currents recorded under voltage clamp from NR1/NR2B receptors that were co-expressed in HEK cells. We applied a brief pulse of a maximally effective concentration of glutamate to outside-out patches that contained only a single active channel. This way, we were able to study the channel response to a single binding event. This approach was combined with analysis of the macroscopic current. From the results of these experiments, we propose a working model of NMDA receptor function that identifies distinct roles for the NR1 and NR2 subunits.

\section{RESULTS}

\section{Determination of NR1/NR2B open probability}

Interpretation of NMDA receptor single-channel recordings has been hampered by the presence of multiple channels in a patch, which adds uncertainty to the origin of closed and open durations. We recorded from outside-out patches that contain a single active NR1/NR2B receptor in response to a brief (1-4 ms) application of a maximally active concentration of glutamate (1 mM; Fig. 1a, top). All solutions contained a saturating concentration of glycine $(20 \mu \mathrm{M})$ unless otherwise stated. Recording the response to a brief pulse of a maximally effective concentration of glutamate ensures that channel openings arise from an individual receptor that binds glutamate once. To identify patches with only one active channel, we recorded the response to brief glutamate application from a large number of patches and counted in each sweep the maximum number of channels that were simultaneously open. We observed several patches with $0,1,2$ or 3 channels that were simultaneously open at any given time. For each patch with only one active channel, we determined the fraction of records with any openings in response to a 1-4 ms application of $1 \mathrm{mM}$ glutamate. Assuming that NR1/NR2B receptors always bind glutamate, then this value corresponds to the probability that an agonist-bound receptor will open at least once before the receptor desensitizes or the agonist unbinds, which we refer to as $P_{\mathrm{o}}$ (burst). We also calculated the open probability at the peak of the response, or $P_{\mathrm{o}}$ (peak), by averaging all records from each patch and then dividing the mean peak current by the single-channel current amplitude (Fig. 1a, bottom). $P_{\mathrm{o}}$ (peak) was $0.17 \pm 0.02$ (mean \pm s.e.m., $n=10$ ) and $P_{\mathrm{o}}$ (burst) was $0.47 \pm 0.06$ $(n=6)$ for patches with only one active channel (Fig. 1b).

Several approaches have been described for determining the number of active channels in a patch ${ }^{12,13}$. Two observations suggest that our recordings were from patches that contained one active channel. First, the open probability at the peak of the response was similar for all patches with a single active channel $\left(P_{\mathrm{o}}\right.$ (peak) range $0.12-0.23$ ). If our sample contained some patches with two or more active channels, we would expect $P_{\mathrm{o}}$ (peak) to vary over a wider range. Second, we predicted that the measured 
a
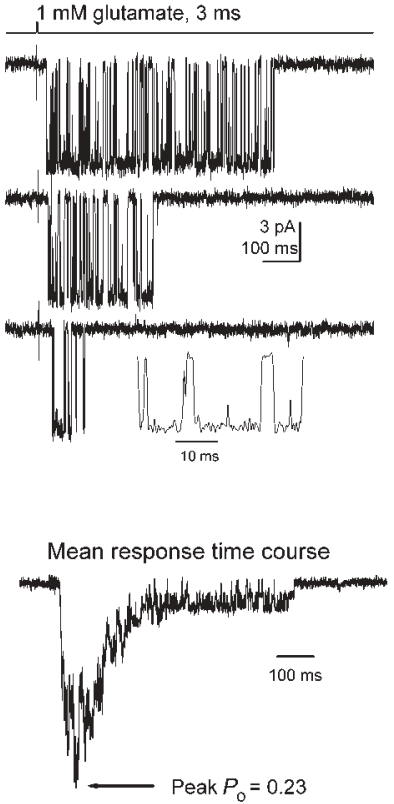

b

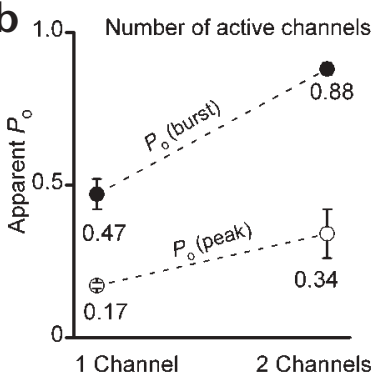

d

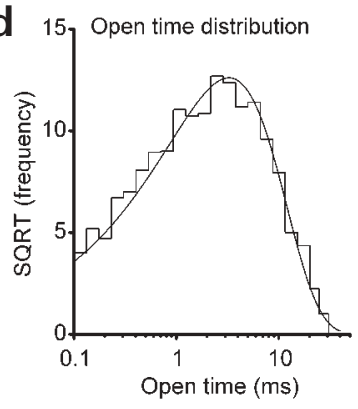

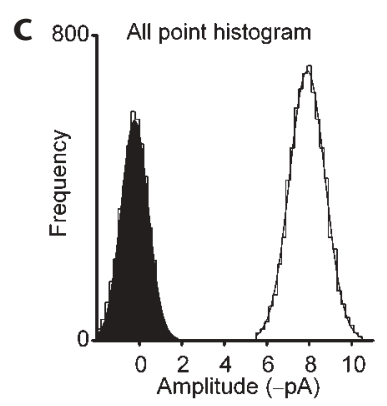

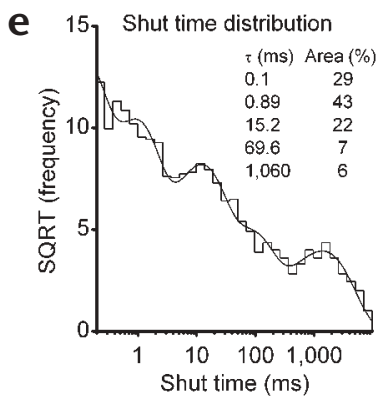

Fig. I. Burst properties of individual NMDA receptor activations. (a) Top, three sweeps are shown from an outside-out patch with a single functional $\mathrm{NRI/NR2B}$ channel $(-100 \mathrm{mV})$ in response to a 4-ms pulse of I mM L-glutamate. Part of the third sweep is expanded (inset). Records are sampled at $13.3 \mathrm{kHz}$ and filtered at I kHz for illustration purposes only; data were filtered at $5 \mathrm{kHz}$ for all analyses. Bottom, the mean response time course of 285 sweeps from the patch in the top panel was divided by the single channel amplitude to determine the peak open probability $\left(P_{\mathrm{o}}\right)$. (b) $P_{\mathrm{o}}$ at the peak of the response (open symbols) was determined as described in (a, bottom) for all patches with either a single active channel or at most two simultaneously open channels. The probability that a channel will open at least once in response to application of maximal concentration of glutamate, $P_{0}$ (burst), is shown for patches with one or two active channels (solid symbols). (c) An all point histogram constructed from a subset of activations for the patch shown in (a, top) was fitted with two Gaussian components $(-7.47 \mathrm{pA}$ and $0.089 \mathrm{pA})$. (d) The composite intra-burst open-time distribution (square root of frequency) for a patch with a single functional NRI/NR2B channel could be well described by a single exponential component $(\tau=3.2 \mathrm{~ms})$. (e) The intra-burst shuttime distribution is shown for the same patch in (d), and could be fitted by five exponential components (inset table shows parameters).

open probability should increase exactly twofold in a patch with at most two channels open simultaneously. Consistent with this prediction, we found that $P_{\mathrm{o}}$ (peak) was increased by a factor of $2.0(0.34 \pm 0.08)$ and $P_{\mathrm{o}}$ (burst) was increased by a factor of 1.9 $(0.88 \pm 0.02)$ in patches with at least one double opening but no triple openings. If some of our apparent single-channel patches contained two or more channels, $P_{\mathrm{o}}$ would increase less than twofold for patches with one double opening. The $P_{\mathrm{o}}$ (peak) value measured here $(0.17)$ is intermediate between the open probability estimated from the progressive MK-801 block of a

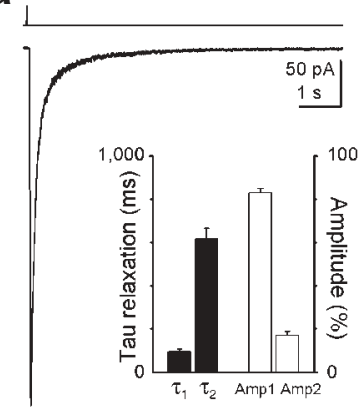

b

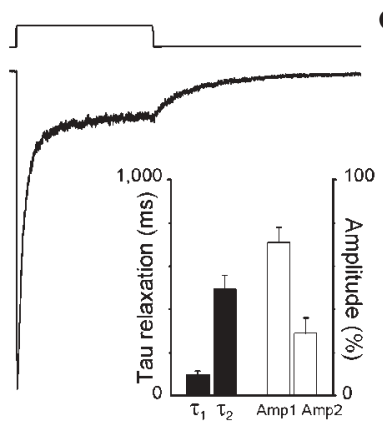

C

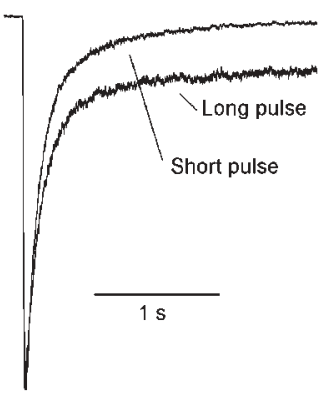

d

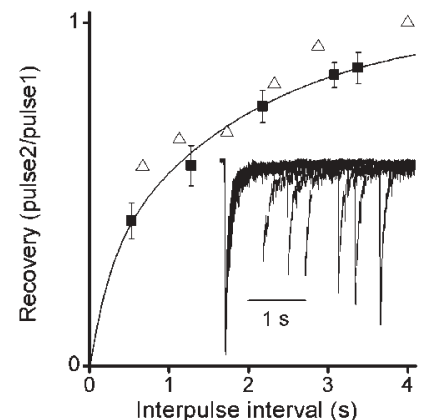

Fig. 2. Macroscopic properties of NRI/NR2B NMDA receptors. Short (a, I-4 ms) or long (b, $3 \mathrm{~s})$ pulses of maximally effective glutamate were applied to outside-out patches expressing NRI/NR2B receptors. The decay current was fitted with two exponential components (Table 2 legend). Fitted time constants for I-4 ms pulses of I mM glutamate were $\tau_{1}=95 \pm \mathrm{II} \mathrm{ms}(83 \pm 2 \%)$ and $\tau_{2}=617 \pm 50 \mathrm{~ms}(17 \pm 2 \%)$. Responses to $3 \mathrm{~s}$ pulses of I mM glutamate had fitted time constants of $\tau_{1}=100 \pm 16 \mathrm{~ms}(7 \mathrm{I}+7 \%)$ and $\tau_{2}=495 \pm 63 \mathrm{~ms}(29 \pm 7 \%) ; n=9$ patches per condition. (c) The normalized current traces from (a) and (b) are shown on an expanded scale. (d) The ratio of the peak responses for pairs of brief glutamate application is plotted against the time interval. Black squares are average data points from nine patches; open triangles are the time course of recovery from desensitization of the patch shown in the insert. Averaged data were fitted with the following equation ${ }^{8}$ : Recovery $=\left(I+\operatorname{Amp} \mid\left[\exp \left(-\right.\right.\right.$ time $\left.\left./ \tau_{1}\right)\right]+$ Amp2[exp $\left(-\right.$ time $\left.\left./ \tau_{2}\right)\right]$ ) where Amp2 $=-I-$ Ampl. 

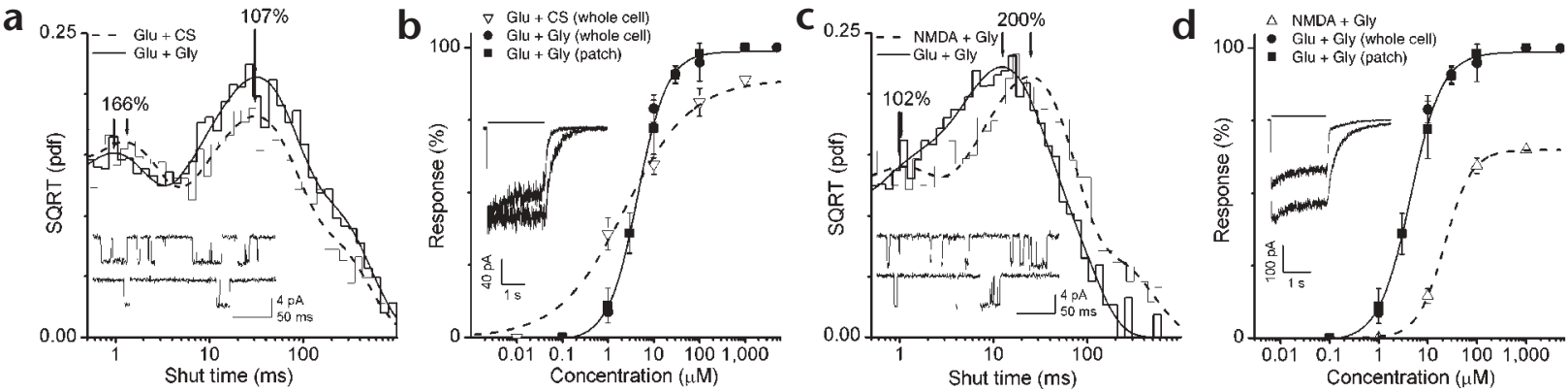

Fig. 3. Subunit-dependent contributions to NMDA receptor gating. (a) The intra-burst shut-time distribution (square root of the probability density function) is shown from one patch with at least two active channels. Maximally effective concentrations of glutamate (I mM) and cycloserine $(0.5 \mathrm{mM})$ were continually applied to the patch (dotted line). Glutamate $(\mathrm{I} \mathrm{mM)}$ and glycine $(20 \mu \mathrm{M})$ were applied as a control before and after CS. The shut time histogram was fitted to four exponential components. The fastest component $(\sim 0.1 \mathrm{~ms})$ is not shown, and may reflect dilation of the pore subsequent to conformational changes in all subunits that are permissive for gating ${ }^{20}$. The fitted components are superimposed onto the histogram. Arrows indicate shifts in fast $(\sim 0.7 \mathrm{~ms})$ and slow $(\sim 13 \mathrm{~ms})$ components (shown as percent change over control). Inset, raw traces from the same patch continuously stimulated with Glu + Gly (upper trace) or Glu + CS (lower trace). Data were sampled at $40 \mathrm{kHz}$ and filtered at $5 \mathrm{kHz}$; imposed resolution was $100 \mu \mathrm{s}$. (b) Responses to variable concentrations NMDA and CS were normalized to the response to maximal glutamate and glycine. $\mathrm{EC}_{50}$ values for dose-response curve were $3.7 \mu \mathrm{M}$ for variable glutamate (+glycine, slope $=1.7, n=6$ patches), $7.6 \mu \mathrm{M}$ for variable glutamate (+glycine, slope $=1.2, n=18$ cells), and $2.1 \mu \mathrm{M}$ for variable CS (+glutamate, slope $=0.4, n=4$ cells). Inset, whole-cell current response to either $100 \mu \mathrm{M}$ glutamate + I mM CS (upper trace) or I mM glutamate $+20 \mu \mathrm{M}$ glycine (lower trace). Cells were held at $-60 \mathrm{mV}$. (c) The intra-burst shut-time distribution is shown from a patch with at least two active channels. A maximal concentration of NMDA $(0.5 \mathrm{mM})$ and glycine $(20 \mu \mathrm{M})$ were continually applied to the patch (dotted line). Glutamate $(\mathrm{I} \mathrm{mM})$ and glycine $(20 \mu \mathrm{M})$ were applied as control before and after NMDA + glycine. (d) EC ${ }_{50}$ values for dose-response curves with variable glutamate (+glycine) are given in (b); the $\mathrm{EC}_{50}$ for variable NMDA was $22.1 \mu \mathrm{M}(+$ glycine, slope $=1.6, n=4$ cells). Inset, whole-cell current response to either I mM NMDA $+20 \mu$ M glycine (upper trace) or I mM glutamate $+20 \mu M$ glycine (lower trace).

NR1/NR2 $\mathrm{B}^{10}$ whole-cell responses (0.07) and of neuronal NMDA receptor responses $(0.3)$ in excised membrane patches from neurons ${ }^{3}$. It has previously been reported that $P_{\mathrm{o}}$ increases in excised patches ${ }^{11}$, suggesting that $P_{\mathrm{o}}$ (peak) values of 0.07 (whole cell) and 0.17 (patch) are compatible.

\section{NR1/NR2B intra-burst closed times}

Our recordings of experimentally defined activations of a single NR1/NR2B receptor allow us to unambiguously define intraburst open and closed lifetimes for a fully liganded receptor. As previously shown ${ }^{5}$, NR1/NR2B channels open primarily to a single conductance state in $0.5 \mathrm{mM} \mathrm{Ca}^{2+}$ (Fig. 1c). The single-channel open-time histogram could be well fitted with a single exponential component with a time constant of $2.9 \pm 0.2 \mathrm{~ms}$ (range 2.0-3.6, $n=6$; Fig. 1d). A second, briefer component was occasionally present ( $n=3$ out of 10 single-channel patches analyzed, mean $0.1 \mathrm{~ms}$ ). We did not further study this glutamate concentration-independent component (data not shown, $n=3$ ) because it is unlikely to impact synaptic transmission at physiological temperatures ${ }^{4,6,7}$.

\section{Macroscopic current properties of NR1/NR2B}

To evaluate the rates of glutamate-induced desensitization, brief (1-4 ms) and long ( $3 \mathrm{~s}$ ) pulses of $1.0 \mathrm{mM} \mathrm{L-glutamate}$ were applied to outside-out patches containing multiple NR1/NR2B channels. Recordings were made in a saturating concentration of glycine, in the presence of $0.5 \mathrm{mM}$ extracellular Ca-EDTA, and in excised patches with $5 \mathrm{mM}$ BAPTA included in the internal solution. These recording conditions minimized the contribution of glycine-dependent desensitization, $\mathrm{Zn}^{2+}$-dependent desensitization and $\mathrm{Ca}^{2+}$-dependent inactivation to the response time course. The macroscopic time course for current decay in the continued presence of glutamate and the current relaxation after brief application of glutamate could be fitted by the sum of two exponential components (Fig. 2a-c). The recovery from desensitization was studied for current responses to brief application of $1 \mathrm{mM}$ glutamate using a double-pulse protocol with a variable time interval. As previously reported ${ }^{8}$, for closely spaced intervals, the peak response amplitude to the second glutamate application was reduced, suggesting that even brief application of agonist can induce desensitization. A plot of the ratio of the peak amplitude of the second response to that of the first response to glutamate versus inter-pulse interval (Fig. 2d) could be fitted by two exponential components ${ }^{8}$. The time constants for recovery from glutamate-induced desensitization were 0.2 and $2.1 \mathrm{~s}(n=9)$.

\section{Do NR1 and NR2 contribute distinct gating steps?}

NMDA receptor kinetics have been notoriously difficult to interpret, partly because of the complexity of the shut-time histogram. The intra-burst shut-time histogram could be fitted with five exponential components (Fig. 1e), as suggested by previous work ${ }^{7}$. We assume that the two slowest components in the intra-burst shut-time histogram ( $\tau=0.070$ and $1.1 \mathrm{~s})$ correspond to the recovery rates from the two desensitized states ( $\tau=0.2$ and $2.1 \mathrm{~s}$ ), as detected by paired-pulse experiments with macroscopic responses in outside-out patches (Fig. 2d). It has previously been suggested $^{14}$ that individual glutamate receptor subunits uniquely contribute to the gating process. Extending this idea, we propose that the time constants describing the three remaining fast components of the shut-time histogram reflect kinetically distinct components of gating. We further propose that these exponential components correspond to agonist-induced conformational changes of either the NR1 or the NR2 subunit, where simultaneous changes in both subunits are required to promote concerted opening of the channel pore (Fig. 4). This idea fits well with the emerging understanding of potassium channel gating based on crystal structures and functional assays ${ }^{15,16}$.

To test this idea, we evaluated the closed-duration histograms in outside-out patches that contain 1-2 channels activated by the continuous application of maximally effective concentrations of both full and partial agonists (Table 1) at either the glycine site on NR1 or the glutamate site on NR2B. Analysis was restricted 

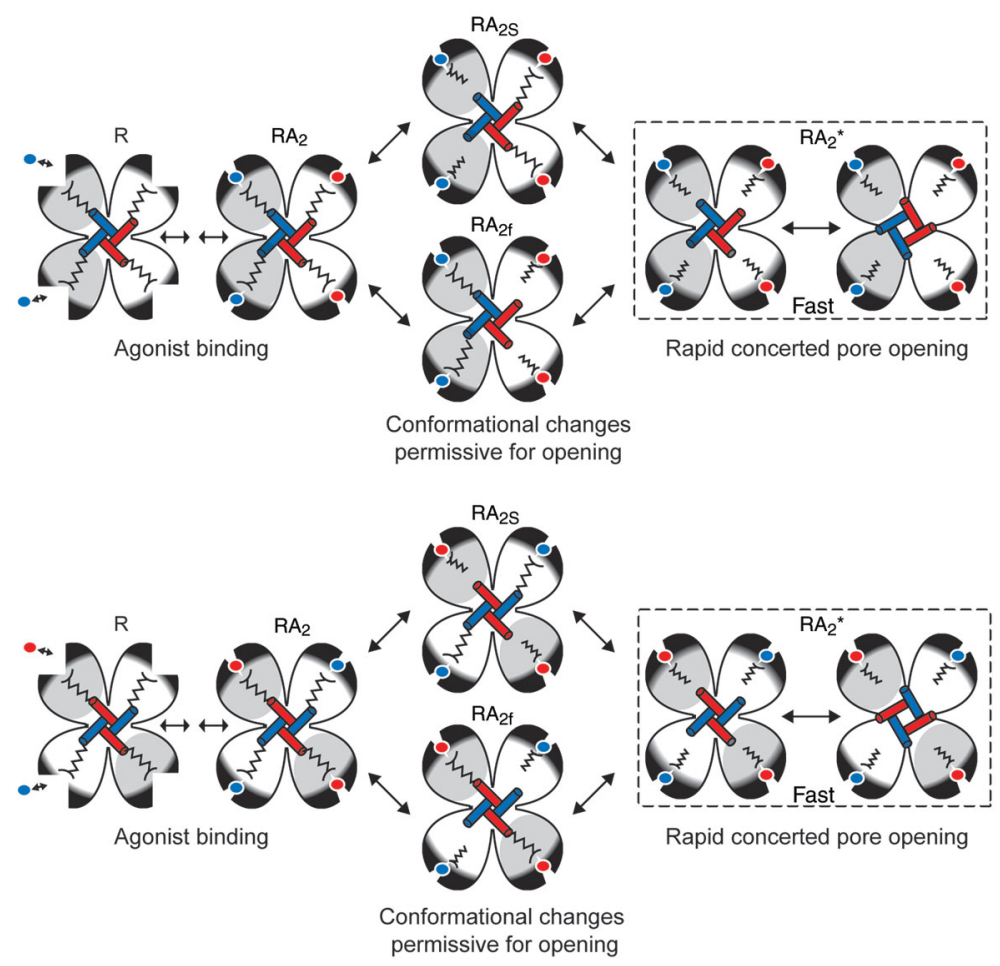

Fig. 4. A model of NMDA receptor activation showing binding and activation of NMDA receptors, incorporating structural ideas about the pore region of the potassium channel $\left.\right|^{31,32}$ and the GluR2 agonist binding pocket ${ }^{22,33}$. Top, a receptor consisting of two dimers ${ }^{17,18,22}$; bottom, a receptor consisting of four subunits in a staggered configuration. In the cartoon, $\mathrm{R}-\mathrm{RA}_{2}$ shows both binding of glutamate and glycine to the receptor and closure of the ligand binding core. $\mathrm{RA}_{2 f}$ and $\mathrm{RA}_{2 \mathrm{~s}}$ show agonist-induced conformational changes in all four subunits, and $\mathrm{RA}_{2} *$ shows rapid concerted opening of the pore. The briefest closures that we observed (mean $\sim 0.1 \mathrm{~ms}$ ) may reflect brief closure of the pore without reversal of any conformational changes that are permissive for gating (for example, movement within states in dotted box). The brief shut-time component ( $0.7 \mathrm{~ms})$ would largely reflect channel movement from state $\mathrm{RA}_{2}{ }^{*}$ to state $\mathrm{RA}_{2 \mathrm{~s}}$, with reversal of an NRI conformational change, followed by reactivation of NRI and pore opening. By analogy, the $13 \mathrm{~ms}$ closure would reflect the same process as the $0.7 \mathrm{~ms}$ closure, except that the active conformation on the NR2 subunit would reverse (move from state $\mathrm{RA}_{2} *$ to state $\mathrm{RA}_{2 f}$ ) and reactivate.

to periods when only one channel was active. We assume that the three fastest shut-time components reflect intra-burst closed periods that are not influenced by random opening and closing of two independent channels. Simulations with two channels in a patch support this assumption (data not shown). Our working hypothesis predicts that a partial agonist would reduce the effective opening rate of the respective subunit's contribution to gating, and thus slow the time constant of the related shut-time component relative to the full agonist. If each subunit controls a kinetically distinct component of gating, we predict that the glycine and glutamate partial agonists will slow different components of the shut-time histogram. Application of glutamate and glycine resulted in histograms that could be fitted by multiple exponential components with time constants that were similar to those for single-channel patches (Fig. 1e), suggesting that meaningful data about the three fastest shut-time components can be extracted from two-channel patches. When the glutamate site partial agonist quinolinic acid (QUIN) or NMDA was applied together with glycine, only one shut-time component (13 ms) was slowed relative to that observed for glutamate + glycine (Fig. 3 and Table 1). On the other hand, when glutamate was applied together with the glycine-site partial agonists cycloserine (CS) or HA-966 (Methods), a different shut-time component (0.7 ms) was slowed compared to that observed with glutamate + glycine (Fig. 3 and Table 1). These data suggest that a rapid glycineinduced conformational change in NR1 and a slower glutamate- induced conformational change in NR2 are both required for opening of the pore. The fastest shut-time component $(0.09 \mathrm{~ms})$ did not vary with partial agonists, suggesting that the briefest shut-time component could reflect the rate for pore dilation once NR1 and NR2 subunits have been activated. As we only detected a single conductance state under our recording conditions, we assume that NMDA pore dilation is a concerted process that requires all subunits to have undergone an agonist-induced conformational change that is permissive for channel opening.

The exponential time constants described here represent complicated expressions of rate constants into and out of several closed states of the channel. However, several control measurements support the idea that NR1 or NR2B conformational changes dominate the two intermediate shut-time components. For example, there were no significant differences between the desensitization rates of current responses activated by either partial or full agonists in multi-channel patches (Table 2). In addition, there were no significant differences in the mean open times (Table 2) observed with any of the partial or full agonists tested. Simulations with models of NMDA receptor activation developed below further support the idea that the transition rates for NR1- and NR2associated conformational changes that precede pore opening dominate the two intermediate time constants (see Fig. 6 legend).

The most parsimonious explanation for these results is that the NR1 and NR2 subunits make fast ( 0.6-0.9 ms) and slow $(\sim 13-30 \mathrm{~ms})$ contributions to the gating process, respectively. 


\begin{tabular}{|c|c|c|c|c|c|}
\hline & $\begin{array}{c}\text { Control } \tau(\mathrm{ms}) \\
\text { (Area, \%) } \\
\text { Glu + Gly }\end{array}$ & $\begin{array}{c}\% \text { of control } \\
\text { (Area, \% of control) } \\
\text { QUIN + Gly }\end{array}$ & $\begin{array}{c}\% \text { of control } \\
\text { (Area, \% of control) } \\
\text { NMDA + Gly }\end{array}$ & $\begin{array}{c}\% \text { of control } \\
\text { (Area, \% of control) } \\
\text { Glu + HA-966 }\end{array}$ & $\begin{array}{c}\% \text { of control } \\
\text { (Area, \% of control) } \\
\text { Glu + CS }\end{array}$ \\
\hline$\tau_{(\mathrm{SHUTI})}$ & $\begin{array}{l}0.09 \pm 0.01 \\
(30 \pm 5)\end{array}$ & $\begin{array}{c}107 \pm 10 \\
(103 \pm 13)\end{array}$ & $\begin{array}{l}100 \pm 4 \\
(96 \pm 21)\end{array}$ & $\begin{array}{l}105 \pm 5 \\
(99 \pm 18)\end{array}$ & $\begin{array}{c}98 \pm 2 \\
(99 \pm 20)\end{array}$ \\
\hline$\tau_{\text {(SHUT2) }}$ & $\begin{array}{l}0.65 \pm 0.06 \\
(16 \pm I)\end{array}$ & $\begin{array}{c}100 \pm 14 \\
(103 \pm 15)\end{array}$ & $\begin{array}{c}102 \pm 17 \\
(104 \pm 15)\end{array}$ & $\begin{array}{l}186 \pm 28 * * \\
(128 \pm 35)\end{array}$ & $\begin{array}{l}167 \pm 20^{* * *} \\
(128 \pm 29)\end{array}$ \\
\hline$\tau_{\text {(SHUT3) }}$ & $\begin{array}{c}13.4 \pm 1.6 \\
(39 \pm 5)\end{array}$ & $\begin{array}{c}208 \pm 24^{* *} \\
(126 \pm 38)\end{array}$ & $\begin{array}{c}196 \pm 29 * \\
(113 \pm 14)\end{array}$ & $\begin{array}{r}|3| \pm 30 \\
(104 \pm 6)\end{array}$ & $\begin{array}{c}114 \pm 14 \\
(105 \pm 11)\end{array}$ \\
\hline
\end{tabular}

Outside-out patches containing one or two NRI/NR2B receptor channels were continually stimulated with either I mM glutamate $(\mathrm{Glu})+20 \mu \mathrm{M}$ glycine (Gly), I mM Glu + 0.5 mM D-cycloserine (CS), I mM Glu + 0.5 mM HA-966, I-5 mM quinolinic acid (QUIN) + $20 \mu M$ Gly or I mM N-methyl-D-aspartate (NMDA) + $20 \mu$ M Gly. Shut-time distributions were fitted with four exponential components; the longest shut-time component, which contains periods during which receptors are in desensitized state(s), is not shown. A resolution of $100 \mu \mathrm{s}$ was imposed on the data; identical results were obtained at $50 \mu \mathrm{s}$ resolution. Only patches with $<17 \%$ change in $\tau$ between control and recovery periods were included. Parameters were normalized for each patch to those determined for glutamate + glycine and shown as percentage of control. $* P<0.05 ; * * P<0.01$ (ANOVA, Bonferroni post hoc test); $n=6-8$ patches. When no significant difference was found, the power to detect a difference of $5 \%$ was 0.86 .

We cannot distinguish whether each shut-time component reflects a single conformational change associated with an NR1 or NR2 dimer ${ }^{17,18}$ (Fig. 4) or independent conformational changes with similar rates on two identical subunits (that is, NR1 or NR2).
Two potential structural changes could reflect the conformational changes that are apparently linked to the intermediate shut-time components. First, kinetically distinct shut-time components could reflect movement of the third membrane-associated region
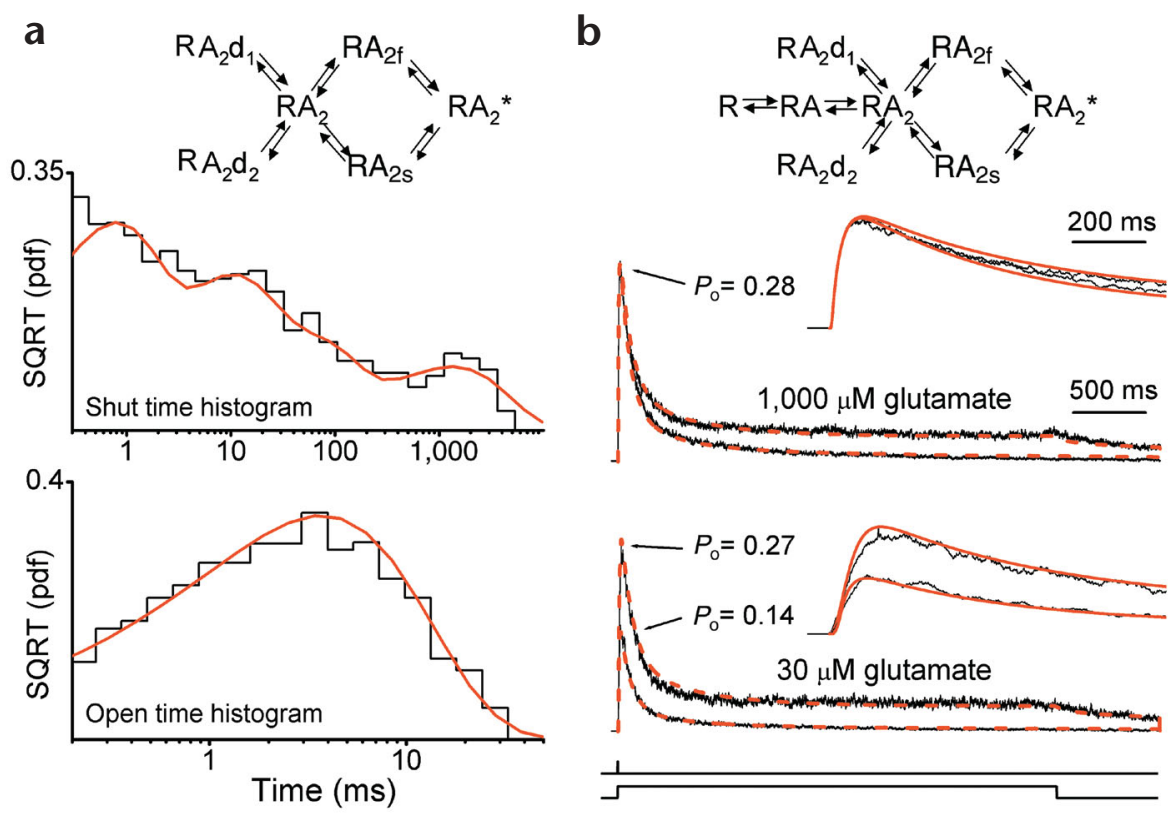

Fig. 5. A physical model of NMDA receptor activation. (a) Top, each NMDA receptor complex is assumed to contain two NRI and two NR2 subunits. Each subunit can independently bind either glycine (NRI) or glutamate (NR2), and the occupancy of the ligand binding domain by agonist eventually leads to a conformational change $(*)$ that is permissive for gating, as shown in Fig. 4. A model illustrating this gating scheme contains a loop, which allows two conformational changes to proceed in any order where $\mathrm{RA}_{2 f}$ and $\mathrm{RA}_{2 s}$ indicate fast and slow conformational changes, respectively, to the active state ${ }^{30,34}$. Two desensitized states are added, as predicted by the dual exponential time course of current response to prolonged agonist application (Fig. 2). The

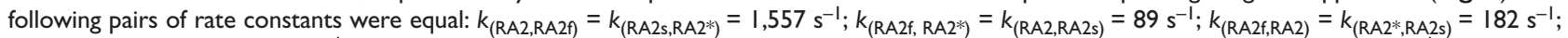
$k_{\left(\text {RA2 }_{2}, \mathrm{RA} 2 \mathrm{f}\right)}=k_{(\mathrm{RA} 2 \mathrm{~s}, \mathrm{RA} 2)}=135 \mathrm{~s}^{-1}$. Rates into desensitization states were allowed to vary during the fitting procedure and $k_{\left(\mathrm{RA} 2, \mathrm{RA}_{2} \mathrm{dI}\right)}=53 \mathrm{~s}^{-1} ; k_{(\mathrm{RA} 2 \mathrm{dI}, \mathrm{RA} 2)}$ $=0.6 \mathrm{~s}^{-1} ; k_{(\mathrm{RA} 2, \mathrm{RA} 2 \mathrm{~d} 2)}=134 \mathrm{~s}^{-1} ; k_{(\mathrm{RA} 2 \mathrm{~d} 2, \mathrm{RA} 2)}=5 \mathrm{~s}^{-1}$. Lower panels are open, and shut-time histograms (square root of the probability density function) predicted from the best maximum likelihood fit ${ }^{29,30}$ to the sequence of open and shut durations (Methods). Measurements from six patches with only one active channel (Fig. I) are superimposed on the composite data. (b) The model (top) was simultaneously fitted to four macroscopic current responses (lower panels) as described in Methods. Only glutamate binding and desensitization rates were allowed to vary during fitting; opening and closing rate constants were fixed to values in Fig. 5a. Fitted time courses ( $3 \mathrm{~s}$ or I-4 ms glutamate applications) are shown as broken red lines. The time course of glutamate application is shown at the bottom. The holding potential was $-60 \mathrm{mV}$. Fitted binding rates were $k_{\text {on }}=9.5 \times 10^{6} \mathrm{M}^{-1} \mathrm{~s}^{-1}\left(\mathrm{RA}, \mathrm{RA} \mathrm{A}_{2}\right)$ and $k_{\text {off }}=29 \mathrm{~s}^{-1}(R A, R)$. We assume that the transition rate for R,RA equals $\left(2 k_{\text {on }}\right)$ and that the rate for $R A_{2}, R A$ equals $\left(2 k_{\text {off }}\right)$. Desensitization rate constants were $k_{(\mathrm{RA} 2, \mathrm{RA} 2 \mathrm{dl})}=45 \mathrm{~s}^{-1}, \mathrm{k}_{(\mathrm{RA} 2, \mathrm{RA} 2 \mathrm{~d} 2)}=70 \mathrm{~s}^{-1}, k_{(\mathrm{RA} 2 \mathrm{~d} 1, \mathrm{RA} 2)}=0.5 \mathrm{~s}^{-1}$ and $k_{(\mathrm{RA} 2 \mathrm{~d} 2, \mathrm{RA} 2)}=2.8 \mathrm{~s}^{-1}$. Inset, the fit of the rising phase on an expanded scale. 
Table 2. Partial NMDA receptor agonist activation and desensitization of NRI/NR2B channels.

\begin{tabular}{lcccccc}
\hline & $\begin{array}{c}\tau_{1} \\
(\mathrm{~ms})\end{array}$ & $\begin{array}{c}\text { Ampl } \\
(\%)\end{array}$ & $\begin{array}{c}\tau_{2} \\
(\mathrm{~ms})\end{array}$ & $\begin{array}{c}\text { Mean open } \\
\text { time }(\mathrm{ms})\end{array}$ & $1 / I_{c}$ & $\mathrm{EC}_{50}(\mu \mathrm{M})$ \\
Glu + Gly & $78 \pm 14$ & $71 \pm 8$ & $580 \pm 28$ & $2.8 \pm 0.3$ & 1 & $4 \pm I(\mathrm{Glu})$ \\
Glu + CS & $98 \pm 32$ & $56 \pm 14$ & $531 \pm 83$ & $2.5 \pm 0.3$ & $0.89 \pm 0.08$ & $2.2 \pm 0.5(\mathrm{CS})$ \\
Glu + HA-966 & $81 \pm 19$ & $58 \pm 15$ & $600 \pm 160$ & $2.6 \pm 0.4$ & $0.81 \pm 0.04$ & $<1(\mathrm{HA}-966)$ \\
NMDA + Gly & $81 \pm 21$ & $79 \pm 5$ & $470 \pm 108$ & $3.1 \pm 0.6$ & $0.65 \pm 0.10$ & $22 \pm 5(\mathrm{NMDA})$ \\
QUIN + Gly & $75 \pm 24$ & $59 \pm 8$ & $715 \pm 170$ & $2.6 \pm 0.2$ & $0.60 \pm 0.13$ & $518 \pm 35(\mathrm{QUIN})$ \\
\hline
\end{tabular}

Outside-out patches expressing NRI/NR2B receptors were stimulated with a $3 \mathrm{~s}$ drug application. Desensitization time course was fitted with: Response = Amp $\left[\exp \left(-\operatorname{time} / \tau_{1}\right)\right]+A m p_{2}\left[\exp \left(-\right.\right.$ time $\left.\left./ \tau_{2}\right)\right]+$ steady state.

The partial agonist concentrations used to determine desensitization properties and mean open time were as shown in Table I. The dose-response relationship was determined from whole-cell recordings from HEK cells expressing NRI/NR2B receptors for the drugs shown in brackets. Composite dose-response curves were fitted with: Response $=$ maximal response/ $\left(I+\left(E_{50} / \text { concentration }\right)^{N}\right)$, where $N$ is the Hill slope.

Concentration-response curves for glycine partial agonists were determined in the presence of $500 \mu \mathrm{M}$ glutamate, and those for glutamate and glutamate partial agonists were determined in the presence of $20 \mu \mathrm{M}$ glycine. EC ${ }_{50}$ for Glu + HA-966 was estimated to be $<\mathrm{I} \mu \mathrm{M}$. There was no significant difference between desensitization properties or mean open times for drugs tested (parametric ANOVA; $n=4-6$ ).

and the linker to ligand binding core, which may precede dilation of the pore ${ }^{19,20}$. Second, each kinetically distinct shut-time component might reflect subunit-specific differences in the rate, extent or stability of the binding domain closure ${ }^{21,22}$. Interestingly, the collision frequency between the two domains that comprise the binding pocket of a bacterial periplasmic galactose-glucose binding protein has been shown to be 10$1,000 \mathrm{~s}^{-1}$ when ligand is bound ${ }^{23}$. Collision rates in this range are compatible with the shut-time components we measure, suggesting that the conformational changes in NR1 and NR2 could be domain closure around the agonist. This seems, however, to be too slow a process to explain the briefest shut-time component, which is about ten times faster $\left(\sim 11,100 \mathrm{~s}^{-1}\right)$.

\section{A physical model of NR1/NR2B receptor function}

Although NMDA receptor burst kinetics are complex, a first approximation of the binding and desensitization rate constants has been obtained using a model with two glutamate binding sites and a single open state ${ }^{8}$. Remarkably good fits have been obtained to the time course of macroscopic currents, suggesting that this model adequately describes glutamate binding, receptor desensitization, and recovery from desensitization. This model does not, however, contain enough closed states to predict intraburst shut times (Fig. 1e). To develop a physical model of NMDA receptor gating that can account for these brief shut-time components as well as macroscopic currents, we modified the single opening step in the previous model $^{8}$ to include subunitdependent gating. We also added a second desensitized state. We illustrate this idea in Fig. 4 by showing independent activation of NR1 and NR2 subunits, assuming that similar subunits behave as a dimer or activate with identical rates. This assumption allows us to use two activation steps. The loop in the model only reflects the idea that NR1 and NR2 conformational changes can occur in any order. We assume that the binding of ligands must take place before any conformational changes occur within a subunit. As our recording solution always contained a saturating concentration of glycine, we assume that NR1 is occupied with glycine at all times and that the glycine rebinding time is negligible. Application of glutamate at a high concentration leads to full occupancy of all four ligand-binding sites, allowing both NR1 and NR2 conformational changes to take place. Finally, we assume a rapid equilibrium between the dilated pore and the closed channel with all four subunits in a conformational state permissive for gating $\left(\mathrm{RA}_{2}{ }^{*}\right)$. We used maximum likelihood fitting of this model to the sequence of single-channel openings from a subset of single-channel patches for which we had recorded a large number (>200) of responses to 1-4 ms application of $1 \mathrm{mM}$ glutamate (Methods). Single-channel records had an imposed resolution of $100 \mu \mathrm{s}$. Figure $5 \mathrm{a}$ shows histograms predicted from the best maximum likelihood fit of this model (without binding steps) to the sequence of intra-burst open and closed intervals from six patches. These data suggest that incorporation of subunit-dependent gating into the previous model $^{8}$ can account for the single channel properties of NR1/NR2B receptors studied here, except for their briefest shut times.

Although the experiments described above provide insight into the burst properties of NMDA receptors, they alone cannot determine the full mechanism of synaptic NMDA receptor activation. To explore whether the model in Fig. $5 \mathrm{~b}$ could explain the time course that we observed in our macroscopic responses from excised outside-out patches containing recombinant NR1/NR2B receptors (Fig. 2), we aligned the recordings from different glutamate concentrations and application durations and averaged response waveforms across patches. We divided all the waveforms by peak amplitude of the responses to $1 \mathrm{mM} \mathrm{L}$-glutamate. A numerical integrator embedded in a simplex algorithm was used to simultaneously fit the model in Fig. $5 \mathbf{b}$ to the four macroscopic response time courses. Opening and closing rates were fixed to those determined from maximum likelihood fitting (Fig. 5a). The close correspondence between measured and fitted waveforms (Fig. 5b) suggests that two glutamate binding sites, two desensitized states and two subunit-specific gating steps are sufficient to account for all of the waveforms that we recorded in response to both brief and prolonged application of glutamate.

To evaluate the predictive potential of this model, we compared simulated current responses to dose-response data (outside-out patches) obtained with short (1-4 ms) and long ( $3 \mathrm{~s}$ ) applications of $0.01,0.03,0.1,0.3$ and $1 \mathrm{mM}$ glutamate. The $\mathrm{EC}_{50}$ of simulated (2.4 $\mu \mathrm{M}$ long pulse, $28 \mu \mathrm{M}$ short pulse $)$ and experimental $(2.8 \mu \mathrm{M}$ short pulse, $30 \mu \mathrm{M}$ long pulse) dose-response curves were in good agreement (Fig. 6a, left). As predicted, simulated long and short application of agonist induced currents with distinct rise times (Fig. 6a, middle). This is because long application of a submaximal concentration of agonist allows the channels to slowly bind agonist. In contrast, brief application of agonist will only allow activation of receptors that happen to bind within 1-4 ms.

If our model is correct to a first approximation, then the open probability predicted from the model for full and partial agonists should qualitatively match our observed recordings. In Fig. 6b and c (right), we show the similarities between observed and pre- 
a

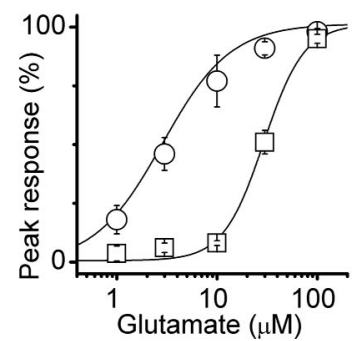

b

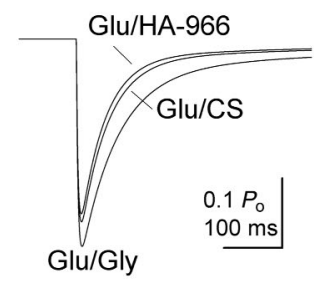

C

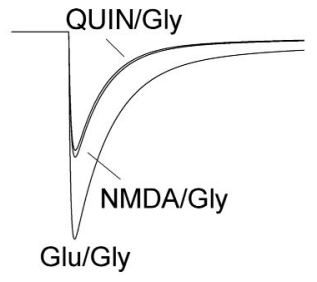

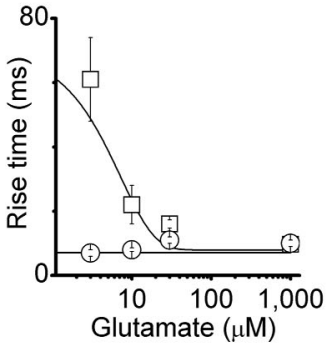
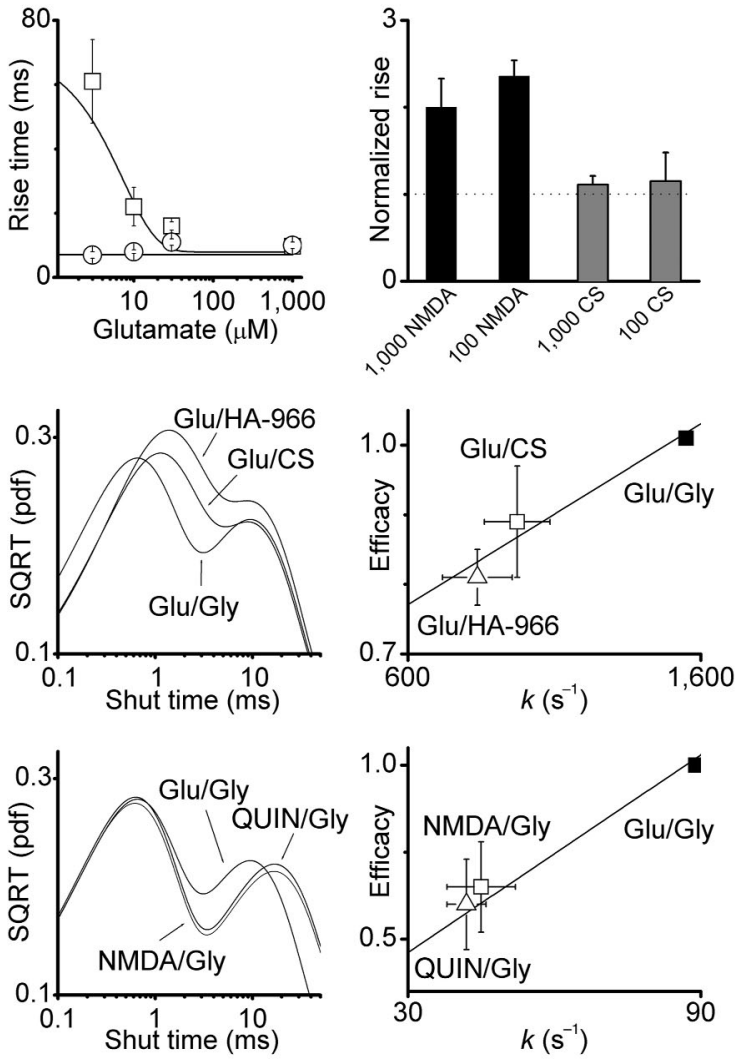

Fig. 6. Simulated responses to glycine and glutamate partial agonists. (a) Left, experimental dose-response relationship for long (circles, $2.8 \mu \mathrm{M}$ ) or short (squares, $30 \mu \mathrm{M})$ pulses of glutamate. Lines show the predicted dose-response relationship for long $(2.4 \mu \mathrm{M})$ or short $(28 \mu \mathrm{M})$ glutamate pulses for the model in Fig. 5b. Middle, measured rise times for short (circles) or long (squares) pulses of glutamate match predicted rise times (lines) from the model shown in Fig. $\mathbf{5 b}$. Right, 10-90\% rise times for responses to NMDA + $20 \mu \mathrm{M}$ glycine and I mM glutamate + CS were normalized to that for I mM glutamate $+20 \mu \mathrm{M}$ glycine (dotted line). Concentration shown is micromolar. (b) Left, simulated macroscopic responses are shown from the model in Fig. 5 with the fast NRI activation rate decreased from I,557 s $\mathrm{s}^{-1}$ (glycine) to $837 \mathrm{~s}^{-1}$ (HA-699) or $932 \mathrm{~s}^{-1}$ (CS). The rate constants associated with NRI activation by glutamate + CS and glutamate + HA-966 were approximated by dividing the fitted activation rate for glutamate by the fold shift in time constants from Table I. Middle, the predicted shut-time histogram shows that only the $0.7 \mathrm{~ms}$ component shifts when the NRI activation rate is slowed. Right, the line shows the relative efficacy predicted when the NRI activation rate varies between $600-1,600 \mathrm{~s}^{-1}$. The response to Glu $+\mathrm{Gly}$ was I.0. Rate constants for glycine, HA-966 and CS activation of NRI are shown as symbols. (c) Left, simulated macroscopic responses are shown from the model in Fig. 5a with the slow NR2 activation rate decreased from $89 \mathrm{~s}^{-1}$ (glutamate) to $45 \mathrm{~s}^{-1}$ (NMDA) or $42 \mathrm{~s}^{-1}$ (QUIN). Rate constants for NR2 activation were estimated as in (b). Middle, the predicted shut-time histogram shows that when the NR2 activation rate is decreased, only the $13 \mathrm{~ms}$ shut-time component is slowed. Right, the line shows the predicted relative efficacy when NR2 activation rate varies between 40-90 $\mathrm{s}^{-1}$. Rate constants for glutamate, NMDA and QUIN activation of NR2 are shown as symbols. There was a linear relationship between $k_{\text {(RA2,RA2f) }}$ (RA2s, RA2*) and $\mathrm{I} / \tau_{\text {shut } 2}$ as well as $k_{\text {(RA2f, RA2*) }}, k_{\text {(RA2,RA2s) }}$ and $\mathrm{I} / \tau_{\text {shut3 }}(R>0.99)$ for the model shown in Fig $5 \mathbf{a}$. The slopes of the normalized rate and shut-time constants $\left(\mathrm{k} / \mathrm{k}_{\text {glu,gly }}\right.$ versus $\left.(1 / \tau) /\left(1 / \tau_{\text {glu,gly }}\right)\right)$ were 0.94 for $\tau_{\text {shut } 2}$ and 0.87 for $\tau_{\text {shut } 3}$, consistent with the idea that specific rate constants dominate the time constants of the two intermediate shut-time components for this model.

dicted relative efficacy from the model in Fig. 5 using the measured values for efficacy and estimations of the subunit specific opening rates (Table 2 and Fig. 5, legends). Our working hypothesis suggests that the two subunit-dependent conformational changes occur before the channel can open. Thus, partial agonists that shift transition rates should have predictable effects on the rise time of macroscopic current responses evoked by supramaximal agonist concentration. For example, the glutamate partial agonist NMDA causes a 12-ms increase in the corresponding shut-time component, and thus should slow the rise time by a comparable amount. In contrast, the glycine site partial agonist CS causes a modest $(0.5 \mathrm{~ms})$ slowing of the $0.7 \mathrm{~ms}$ shut-time component, which is unlikely to cause a detectable change in rise time. Consistent with these predictions, the glutamate partial agonist NMDA slowed the rise time approximately twofold (14.4 $\pm 2.2 \mathrm{~ms}$ for glutamate versus $27.8 \pm 4.7 \mathrm{~ms}$ for NMDA, $n=4$; $P>0.05)$, whereas no significant shift in the rise time could be detected for the glycine site partial agonist CS (13.7 \pm 1.6 for glycine versus $14.7 \pm 1.25$ for CS, $n=4$; Fig. 6a, right).

\section{Discussion}

By analyzing patches that contain only a single receptor and activating these receptors with brief application of a maximal glutamate concentration, we were able to obtain an unambiguous description of single-channel properties within a single activation of an NMDA receptor with defined subunit composition. Evaluation of these data together with macroscopic response time courses has allowed us to propose a unifying hypothesis for the activation of NMDA receptors that centers on the idea of kinetically distinct contributions of NR1 and NR2 subunits to channel 
opening (Figs. 4 and 5). This model is an extension of a previous hypothesis describing NMDA receptor function, yet it embodies novel representation of unique NR1 and NR2 subunit function. Our incorporation of subunit-dependent steps in the gating scheme gives the working model a strong physical base. For example, this model allows us to interpret the shut-time histogram in terms of physical events. Each of the shut-time components we observed (Fig. 1e) started with closure of the pore. The briefest component could reflect rapid reopening of the channel, whereas the intermediate closed states involve a subsequent reversal of an NR1 or NR2 conformational change that is permissive for gating. These conformational changes could give the 0.7 (NR1) or 13 (NR2) ms closed time components. The two slowest shut-time components involve movement into a desensitized state and subsequent slow recovery and reactivation.

Although our model can account for all of the data we have obtained, there are nevertheless several limitations that suggest future experimental directions. For example, improved recording resolution will undoubtedly reveal more exponential components within the shut and open-time histograms, which will need to be accounted for by revisions of our working hypothesis. In addition, we have used recording conditions that markedly reduce the relative contribution of the $40 \mathrm{pS}$ subconductance level, allowing open times to be described by a single exponential component. Revisions of our model will be necessary to fully account for NMDA receptor function in neurons that include subconductance levels. Furthermore, patch recordings show exaggerated desensitization, suggesting that more work is needed to better understand the effects of desensitization on native synaptic and extrasynaptic NMDA receptors. Finally, we do not yet have a physical hypothesis underlying the dual exponential time course of desensitization.

Yet despite these shortcomings, our findings represent an important step forward in understanding NMDA receptor function. Our model is the first that can reconcile microscopic and macroscopic properties of channel function. In addition, our analysis provides an example of how different subunits within the same receptor complex can uniquely contribute to complex channel properties, and thus holds important implications for a wide range of heteromeric, ligand-gated channels. Furthermore, the different activation rate of NR1 and NR2 subunits is consistent with previous reports of asymmetrical gating ${ }^{20,24}$. This idea has a strong physical basis in that the two kinetically distinct gating elements represent different receptor subunits. Such insight should facilitate both understanding of the structural features of channel gating and serve as a starting point for studies of subunit-specific modulation of NMDA receptors by phosphorylation or by extracellular allosteric regulators.

\section{MethodS}

Cell culture. HEK 293 cells were maintained as previously described ${ }^{25}$ and plated onto $12 \mathrm{~mm}$ glass coverslips that were coated with poly-Dlysine (5-10 $\mu \mathrm{g} / \mathrm{ml})$. cDNAs encoding NR1-1a (GenBank U08261) and NR2B (U11419) in pcIneo or pcDNA1/amp were transiently transfected into cells using the calcium-phosphate precipitation method ${ }^{26}$. The ratio of cDNAs encoding NR1:NR2:green fluorescent protein (GFP) for experiments with single-channel patches was 1:2:12 with a final NR1 cDNA concentration of $0.08 \mu \mathrm{g} / \mathrm{ml}$. To obtain larger macroscopic currents, we used a transfection ratio of 1:1:1.5 for NR1:NR2B:GFP and a final concentration of $0.5 \mu \mathrm{g} / \mathrm{ml} \mathrm{NR} 1 \mathrm{cDNA}$.

Electrophysiology. Cells remained in the transfection solutions for $2-5 \mathrm{~h}$ for single-channel patch experiments and 6-8 $\mathrm{h}$ for macroscopic experiments, after which the medium was replaced and supplemented with 2-3 $\mathrm{mM} \mathrm{Mg}^{2+}$ and $200 \mu \mathrm{M}$ DL-2-amino-5-phosphonopentanoic acid.
Recordings were typically made over the next $24 \mathrm{~h}$. Glutamate was applied using a piezoelectric translator (Burleigh, Victor, New York) to move a double-barreled flow pipe constructed from theta tubing (Hilgenberg, Malsfeld, Germany). As previously described ${ }^{27}$, pipette tip potentials were measured after each experiment; $10-90 \%$ rise times were between $0.3-0.6 \mathrm{~ms}$. A triple glass flow pipe was used for the partial agonist experiments. Each barrel was continually superfused with the same drugs during the same day to limit any possible cross-contamination. External recording solution for all experiments consisted of $150 \mathrm{mM} \mathrm{NaCl}, 10$ mM HEPES, $3 \mathrm{mM} \mathrm{KCl}, 0.7 \mathrm{mM} \mathrm{CaCl}_{2}$ and $0.2 \mathrm{mM}$ EDTA; $\mathrm{pH} 7.4$ $(\mathrm{NaOH}), 0.31-0.33$ osM. The reduction of free extracellular $\mathrm{Ca}^{2+}$ to 0.5 $\mathrm{mM}$ reduced the frequency of subconductance levels ${ }^{5}$; EDTA was added to remove any contaminant divalent cations. Evaluation of the $\mathrm{pH}$ sensitivity indicated that there was $13.5 \pm 0.1 \%$ ( $n=5$ patches) tonic proton inhibition under our recording conditions. All experiments were performed at $23.2 \pm 0.2^{\circ} \mathrm{C}$. The internal solution consisted of $110 \mathrm{mM}$ gluconic acid, $30 \mathrm{mM} \mathrm{CsCl}, 4 \mathrm{mM} \mathrm{NaCl}, 5 \mathrm{mM}$ HEPES, $5 \mathrm{mM}$ BAPTA, $0.5 \mathrm{mM} \mathrm{CaCl}_{2}$ and $2 \mathrm{mM} \mathrm{MgCl}_{2} ; \mathrm{pH} 7.3(\mathrm{CsOH}), 0.29-0.30$ osM. Glutamate was applied at $3,30,100,300$ or $1,000 \mu \mathrm{M}$ for either $1-4 \mathrm{~ms}$ or 3 s. All solutions contained a maximal concentration of glycine $(20 \mu \mathrm{M}$ or $\left.\sim 50 \times \mathrm{EC}_{50}\right)$ and glutamate $(1 \mathrm{mM})$ except for dose-response experiments and experiments in which the following partial agonists were used: $(+)-$ 3-amino-1-hydroxypyrrolidin-2-one (HA-966; $0.5 \mathrm{mM}$ ), D-cycloserine (CS; $0.5 \mathrm{mM}$ ), $N$-methyl-D-aspartate (NMDA; $0.5 \mathrm{mM}$ ) and quinolinic acid (QUIN; 1-5 mM). Thick-walled borosilicate glass (OD 1.5, ID 0.85 $\mathrm{mm}$; Warner Instruments, Hamden, Connecticut) was fire-polished to a resistance of 6-9 $M \Omega$, and sylgard was applied to the pipette tip. All agonists and antagonists except glutamate were obtained from TocrisCookson (Ellisville, Missouri). Glutamate and other chemicals were from Sigma (St. Louis, Missouri).

Data analysis. Data are shown as the mean \pm standard error of mean (s.e.m.). Single-channel and macroscopic data were digitized at 13-40 kHz using Clampex v8.0 (Axon Instruments, Union City, California) after filtering at $5 \mathrm{kHz}$ (8-pole Bessel, $-3 \mathrm{~dB}$, Frequency Devices, Haverhill, Massachusetts). Data for some experiments were first stored on DAT tape (Dagan, Minneapolis, Minnesota) at $48 \mathrm{kHz}$. Multiple macroscopic waveforms were divided by the peak amplitude of the maximum current response and fitted with a simplex algorithm (least squares criteria) that modified kinetic rate constants as free variables. Simulated waveforms at each step in the simplex were obtained by numerical integration (Channelab, Synaptosoft, Decatur, Georgia). At each iteration, the peak of the maximal response was scaled to the peak open probability of the simulated response, and all other waveforms were scaled relative to the maximum peak response. Single-channel records were analyzed by time-course fitting with SCAN (provided by D. Colquhoun, University College London, UK) or using the Viterbi algorithm in QUB Software (www.qub.buffalo.edu). Open and closed duration histograms were fitted with multiple exponential components in Channelab using the maximum likelihood method ${ }^{28}$ with a resolution of $100 \mu$ s. Maximum likelihood fits of the sequence of open and shut intervals within individual activations were performed as previously described $^{29,30}$ using MIL (QUB software, Buffalo, New York). Fitting of the sequence of open and closed durations was performed (first to last open time) on records pooled from six experiments ( $n=1,535$ activations), with initial occupancy set to be in the open state. Amplitude histograms were determined from QUB, SCAN and MiniAnalysis (Synaptosoft) software.

\section{Acknowledgments}

We thank S. Heinemann for sharing NMDA receptor cDNAs and D. Colquhoun for helpful discussions and sharing of software. In addition, we thank T. Auerbach for helpful discussions, critical comments on the manuscript and for suggesting the experiments with partial agonists. We also thank K. Erreger,

A. Gibb and S. Cull-Candy for providing helpful comments on the manuscript. This work was supported by an Alfred Benzon Foundation grant (T.B.), a Danish Medical Research Fellowship (T.B.) and the National Institutes of Health (NS36654, S.F.T.). 


\section{Competing interests statement}

The authors declare that they have no competing financial interests.

\section{ReCeived 1 November; ACCEPTEd 19 DeCember 2002}

1. Dingledine, R., Borges, K., Bowie, D. \& Traynelis, S.F. The glutamate receptor ion channels. Pharmacol. Rev. 51, 7-61 (1999).

2. Lester, R.A., Clements, J.D., Westbrook, G.L. \& Jahr, C.E. Channel kinetics determine the time course of NMDA receptor-mediated synaptic currents. Nature 346, 565-567 (1990).

3. Jahr, C.E. High-probability opening of NMDA receptor channels by Lglutamate. Science 255, 470-472 (1992).

4. Edmonds, B. \& Colquhoun, D. Rapid decay of averaged single-channel NMDA receptor activations recorded at low agonist concentration. Proc. $R$. Soc. Lond. B Biol. Sci. 250, 279-286 (1992).

5. Premkumar, L.S., Qin, F. \& Auerbach, A. Subconductance states of a mutant NMDA receptor channel kinetics, calcium and voltage dependence. J. Gen. Physiol. 109, 181-189 (1997).

6. Anson, L.C., Schoepfer, R., Colquhoun, D. \& Wyllie, D.J. Single-channel analysis of an NMDA receptor possessing a mutation in the region of the glutamate binding site. J. Physiol. 527, 225-237 (2000).

7. Gibb, A.J. \& Colquhoun, D. Activation of N-methyl-D-aspartate receptors by L-glutamate in cells dissociated from adult rat hippocampus. J. Physiol. 456, 143-179 (1992).

8. Lester, R.A. \& Jahr, C.E. NMDA channel behavior depends on agonist affinity. J. Neurosci. 12, 635-643 (1992)

9. Schneggenburger, R. \& Ascher, P. Coupling of permeation and gating in an NMDA-channel pore mutant. Neuron 18, 167-177 (1997).

10. Chen, N., Luo, T. \& Raymond, L.A. Subtype-dependence of NMDA receptor channel open probability. J. Neurosci. 19, 6844-6854 (1999).

11. Rosenmund, C., Feltz, A. \& Westbrook, G.L. Synaptic NMDA receptor channels have a low open probability. J. Neurosci. 15, 2788-2795 (1995).

12. Colquhoun, D. \& Hawkes, A.G. Stochastic properties of ion channel openings and bursts in a membrane patch that contains two chanels: evidence concerning the number of channels present when a record containing only single openings is observed. Proc. R. Soc. Lond. B Biol. Sci. 240, 453-477 (1990).

13. Horn, R. Estimating the number of channels in patch recording. Biophys. J. 60, 433-439 (1991).

14. Rosenmund, C., Stern-Bach, Y. \& Stevens, C.F. The tetrameric structure of a glutamate receptor channel. Science 280, 1596-1599 (1998).

15. Perozo, E. New structural perspectives on $\mathrm{K}^{+}$channel gating. Structure 10, 1027-1029 (2002).

16. Yellen, G. The voltage-gated potassium channels and their relatives. Nature $419,35-42(2002)$.
17. Mansour, M., Nagarajan, N., Nehring, R.B., Clements, J.D. \& Rosenmund, C. Heteromeric AMPA receptors assemble with a preferred subunit stoichiometry and spatial arrangement. Neuron 32, 841-853 (2001).

18. Antoine R., Stacey N.I., Hughes, T.E. \& Howe, J.R. Subunit interactions and AMPA receptor desensitization. J. Neurosci. 21, 5574-5586 (2001).

19. Sobolevsky, A.I., Beck, C. \& Wollmuth, L.P. Molecular rearrangements of the extracellular vestibule in NMDAR channels during gating. Neuron 33, 75-85 (2002).

20. Jones, K.S., VanDongen, H.M.A. \& VanDongen, A.M.J. The NMDA receptor M3 segment is a conserved transduction element coupling ligand binding to channel opening. J. Neurosci. 22, 2044-2053 (2002).

21. Benveniste, M. \& Mayer, M.L. Trapping of glutamate and glycine during open channel block of rat hippocampal neuron NMDA receptors by 9aminoacridine. J. Physiol. 483, 367-385 (1995).

22. Sun, Y. et al. Mechanism of glutamate receptor desensitization. Nature 417, 245-253 (2002).

23. Careaga, C.L., Sutherland, J., Sabeti, J. \& Falke, J.J. Large-amplitude twisting motions of an interdomain hinge: a disulfide trapping study of the galactose-glucose binding protein. Biochemistry 34, 3048-3055 (1995).

24. Sobolevsky, A.I., Rooney, L. \& Wollmuth, L.P. Staggering of subunits in NMDAR channels. Biophysical. J. 83, 3304-3314 (2002).

25. Traynelis, S.F. \& Wahl, P. Control of rat GluR6 glutamate receptor open probability by protein kinase A and calcineurin. J. Physiol. 503, 513-531 (1997).

26. Chen., C. \& Okayama, H. High-efficiency transformation of mammalian cells by plasmid DNA. Mol. Cell. Biol. 7, 2745-2752 (1987).

27. Banke, T.G. et al. Control of GluR1 AMPA receptor function by cAMPdependent protein kinase. J. Neurosci. 20, 89-102 (2000).

28. Colquhoun, D. \& Sigworth, F.J. Fitting and statistical analysis of singlechannel records. in Single-channel Recording $2^{\text {nd }}$ edn. (eds. Sakmann, B. \& Neher, E.) 483-585 (Plenum Press, New York, 1995).

29. Qin, F., Auerbach, A. \& Sachs, F. Estimating single-channel kinetic parameters from idealized patch clamp data containing missed events. Biophysical J. 70, 264-280 (1996).

30. Qin, F., Auerbach, A. \& Sachs, F. Maximum likelihood estimation of aggregated Markov processes. Proc. R. Soc. Lond. B Biol. Sci. 264, 375-383 (1997).

31. Perozo, E., Cortes, D. M. \& Cuello, L.G. Structural rearrangements underlying K+-channel activation gating. Science 285, 73-78 (1999).

32. Jiang, Y. et al. The open pore conformation of potassium channels. Nature 417, 523-526 (2002).

33. Armstrong, N. \& Gouaux, E. Mechanisms for activation and antagonism of an AMPA-sensitive glutamate receptor: crystal structures of the gluR2 ligand binding core. Neuron 28, 165-181 (2000).

34. Akk, G., Zhou, M. \& Auerbach, A. A mutational analysis of the acetylcholine receptor channel transmitter binding site. Biophys. J. 76, 207-218 (1999). 